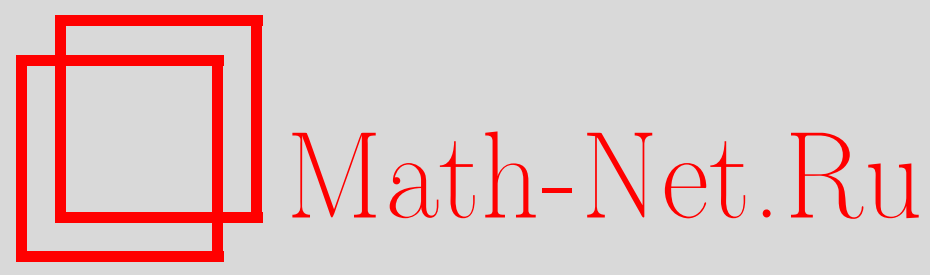

Н. С. Гусев, О выпуклых реализациях плоских линейных деревьев, Матем. заметки, 2003, том 73, выпуск 5, 665675

DOI: https://doi.org/10.4213/mzm218

Использование Общероссийского математического портала Math-Net.Ru подразумевает, что вы прочитали и согласны с пользовательским соглашением http://www . mathnet.ru/rus/agreement

Параметры загрузки:

IP : 44.207 .124 .84

26 апреля 2023 г., 12:58:37

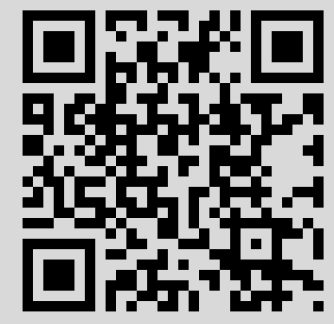




\title{
О ВЫПУКЛЫХ РЕАЛИЗАЦИЯХ ПЛОСКИХ ЛИНЕЙНЫХ ДЕРЕВЬЕВ
}

Н. С. Гусев

\begin{abstract}
В настоящей статье доказан необходимый признак существования выпуклой реализации у плоского линейного дерева; установлено, что этот признак достаточен для случая ломаных и найден непрерывный алгоритм построения такой реализации.

Библиография: 7 названий.
\end{abstract}

1. Введение. В настоящей работе изучается задача вьпуклой реализации плоских линейных деревьев. Это одна из задач, связанных с известной проблемой Штейнера (см. [1]-[4]) поиска сети минимальной длины, затягивающей данное конечное множество точек на плоскости; это множество обычно называется граничны. .

В связи с тем, что проблема Штейнера является NP-трудной (см. [1]-[5]), А. О. Ивановым и А. А. Тужилиным в [2] была поставлена задача о зависимости между геометрией гранищы и возможными типами (топологиями) минимальной сети. Один из подходов к изучению этой зависимости - рассматривать параллельные реализации (определение см. ниже) линейных плоских бинарных деревьев с межреберными углами при вершинах равными $2 \pi / 3$, при том, что граничное множество обладает некоторыми заданными геометрическими свойствами; например, оно расположено на границе вьпуклого многоугольника. В качестве обобщения этой задачи можно рассмотреть параллельные реализации взвешенных локально минимальных деревьев (см. [6], [7]) и, далее, произвольных деревьев.

Прежде чем сформулировать задачу и результат этой работы, приведем некоторые определения, см. также [6].

Полуплоскость $P$ вместе с некоторой частью своей границы назовем опирающейся на точку $p$, если $p \in \partial P$.

Вложением связного неориентированного без кратных ребер и петель графа $G=$ $(V, E)$ в плоскость назовем такое инъективное отображение $\tau$, определенное на $V \sqcup E$ и сопоставляющее вершинам точки, а ребрам интервалы, что замыкания образов несмежных ребер непересекаются, а замыкания образов смежных ребер пересекаются только по образу их общей вершины. Вложение $\tau$ графа $G$ будем также обозначать $(G, \tau)$, или говорить, что граф $G \tau$-вложсенный. Значение вложения $\tau$ графа $G$ на вершине $p$ будем обозначать $\tau_{p}$ или $\tau(p)$.

Вершину $p \tau$-вложенного графа $G$ назовем $\tau$-граничной (әраничной), если найдется открытая полуплоскость $P$, опирающаяся на образ $p$, такая, что все $\tau$-образы ребер графа $G$, инцидентных этой вершине, лежат в $P$; неграничную вершину такую, что образы всех инцидентных ей ребер располагаются на одной прямой, назовем $\tau$-фиктивной. 
Множество всех $\tau$-граничных вершин графа $G$ назовем его $\tau$-границей (границей) и обозначим через $\delta_{\tau} G$.

Два вложения графов $(G, \alpha)$ и $(H, \beta)$ назовем параллельными, если найдутся изометрия $\psi$ плоскости $\mathbb{R}^{2}$ и изоморфизм $\phi$ графов $G$ и $H$ такие, что для произвольных трех вершин $p, q, r$ графа $G$ таких, что $p$ и $r$ соседние с $q$, вьполняется равенство

$$
\angle\left(\overrightarrow{\alpha_{q} \alpha_{p}}, \overrightarrow{\alpha_{q} \alpha_{r}}\right)=\angle\left(\overrightarrow{\gamma_{q} \gamma_{p}}, \overrightarrow{\gamma_{q} \gamma_{r}}\right)
$$

где $\gamma=\psi \circ \beta \circ \phi-$ композиция отображений, а $\angle(a, b)$ - ориентированный угол от вектора $a$ до вектора $b$, принимающий значения в $(-\pi, \pi]$.

Вложение графа назовем выпуклы.м, если его граница лежит на границе вьпуклого многоугольника, и выпукло реализуемым, если найдется ему параллельное выпуклое вложение.

Пусть $(T, \tau)$ - вложение дерева, $p \in \delta_{\tau} T$.

Тогда ребро $e$, инцидентное $p$, назовем $p$-опорным, если образы всех ребер, инцидентных $p$, лежат в одной замкнутой полуплоскости относительно прямой, проходящей через образ е.

Заметим, что у всякой граничной вершины $p$ или одно или два опорных ребра. Если их два, то занумеруем их $\left(e_{1}^{p}, e_{2}^{p}\right)$ так, чтобы пара векторов направлений этих ребер с началами в $р$ образовывала положительно ориентированньй базис. Если опорное ребро одно, то положим $e_{1}^{p}=e_{2}^{p}$.

Назовем фрагментом дерева $T$, соответствующим ребру е (фрагментом ребра $e)$, максимальньй по включению отрезок среди всех отрезков, содержащихся в образе дерева $T$ и содержащих образ ребра $e$.

Ребро е назовем двусторонним, если среди образов ребер, пересекающих фрагмент ребра $e$, найдутся два, лежашие по разные стороны относительно прямой, проходящей через этот фрагмент.

Назовем ломаной дерево с не менее чем одним ребром, степени всех вершин которого суть 1 или 2. Простой ломаной будем называть образ вложения ломаной. Ломаная ориентирована, если известно (задано) какая из двух ее вершин степени один начальная и какая концевая. У всякой ориентированной ломаной $A$ обозначим $A_{0}, \ldots, A_{n+1}$ все ее вершины в порядке ориентации, а число $n$ назовем длиной ломаной. Для вложения $f$ ориентированной ломаной $A$ длины $n>0$ обозначим

$$
\begin{aligned}
& \operatorname{ang}_{f}(i):=\angle\left(\overrightarrow{f\left(A_{i-1}\right) f\left(A_{i}\right)}, \overrightarrow{f\left(A_{i}\right) f\left(A_{i+1}\right)}\right), \quad i=1, \ldots, n \\
& \operatorname{ang}_{f}:=\left(\operatorname{ang}_{f}(1), \ldots, \operatorname{ang}_{f}(n)\right), \quad \operatorname{tw}(f):=\sum_{i=1}^{n} \operatorname{ang}_{f}(i)
\end{aligned}
$$

Заметим, что иногда сумму в tw делят на $\pi / 3$ (см. [6], [7]).

Пусть $(T, \tau)$ - вложение дерева, $p, q \in \delta_{\tau} T, p \neq q$. Тогда введем обозначения

- $T^{p q}$ - ориентированная от $p$ к $q$ ломаная, соединяющая вершины $p$ и $q$ в дереве $T$;

- $\widehat{T}^{p q}$ - ломаная, получаемая из $T^{p q}$ добавлением тех ребер из $e_{1}^{p}$ и $e_{2}^{q}$, которые не входят в $T^{p q}$, с сохранением ориентации ломаной. 
Обозначив $\omega$ вложение ломаной $\widehat{T}^{p q}$, осуществляемое вложением всего дерева, определим также следующую функцию:

$$
\operatorname{agg}_{\tau}(p, q):=\operatorname{tw}(\omega)-\nu \pi
$$

где $\nu$ - количество тех ребер из набора $\left(e_{1}^{p}, e_{2}^{q}\right)$, которые входят в состав ломаной $T^{p q}$.

Назовем вложение $(T, \tau)$ строго неположительным, если для всяких неравных граничных вершин $p, q$ выполняются оба условия

1. $\operatorname{agg}_{\tau}(p, q) \leqslant 0$

2. если хотя бы одно из ребер $e_{1}^{p}$ или $e_{2}^{q}$ двустороннее, то неравенство строгое.

ЗАДАчА. Исследовать, при каких условиях вложение дерева является вьпукло реализуемьм.

ТеОрема 1.1. Если вложсение дерева $(T, \tau)$ выпукло реализуемо, то оно строго неполохительно.

ТЕОремА 1.2. Вложсение ломаной $(T, \tau)$ выпукло реализуемо, если и только если оно строго неположительно.

Кроме того, найден алгоритм построения выпуклой реализации и описано геометрическое строение совокупности всех классов параллельности вьпукло реализуемых ломаных.

2. Общее необходимое условие выпуклой реализуемости вложений деревьев. В этом разделе мы докажем теорему 1.1. Ее доказательство проведем в пяти леммах. Будем считать, что образ дерева не является отрезком, так как в случае, когда образ дерева - отрезок, эта теорема очевидна.

Прежде всего определим некоторые понятия. Пусть $(F, f),(G, g)$ - вложения ориентированных ломаных. Тогда обозначим:

- $A:=f(F) \cap g(G)$;

- $B$ - совокупность связных компонент множества $A$ (заметим, что этих связных компонент конечное число и каждая из них является или точкой или простой ломаной);

- для каждой связной компоненты $b \in B$ обозначим через $\hat{b}$ совокупность концевых точек $b$;

- $C:=\bigcup_{b \in B} \hat{b}$ (ясно, что $C$ - конечное подмножество в $A$ );

- упорядочив $C$ вдоль $f$ и $g$, получим соответственно $\mu=\left(\mu_{1}, \ldots, \mu_{n}\right)$ и $\nu=$ $\left(\nu_{1}, \ldots, \nu_{n}\right)$ - конечные последовательности всех точек из $C$ такие, что $\mu_{i}$ предшествует $\mu_{i+1}$ вдоль $f$, и $\nu_{i}$ предшествует $\nu_{i+1}$ вдоль $g$.

Лемма 2.1. Пусть $(F, f),(G, g)$ - такие вложсния ориентированных ломаных, что их начальные и конечные точки совпадают и существует односвязный многоугольник $M$, содержащий в себе $g$, частью гранииы которого является ломаная $f$. Тогда $\mu=\nu$.

ДокАЗАТЕЛЬСтво. Прежде всего заметим, что $\mu_{1}=\nu_{1}$ и $\mu_{n}=\nu_{n}$.

Допустим $\mu \neq \nu$; тогда $i:=-1+\min \left\{j: \mu_{j} \neq \nu_{j}\right\}$ и найдется $j: \nu_{i+1}=\mu_{j}$, а значит, $j>i+1$.

Обозначим $\tilde{f}$ участок образа ломаной $f$ от $\mu_{i}$ до $\mu_{j}$, а $\tilde{g}$ - участок образа ломаной $g$ от $\nu_{i}$ до $\nu_{i+1}$. 
1. Заметим, что если бы $A \supset \tilde{g}$, то $\nu_{i}$ и $\nu_{i+1}$ являлись бы концевыми точками ломаной $\tilde{g}$, а значит, $\tilde{g} \in B$; но тогда $\mu_{i}$ и $\mu_{j}$ были бы соседними точками $C$ вдоль $f$, т.е. $i+1=j$, что противоречит предположению. Следовательно, $A \not \supset \tilde{g}$.

2. Если $\tilde{f} \cap \tilde{g} \neq\left\{\mu_{i}, \mu_{j}\right\}$, т.е. существует $\alpha \in \tilde{f} \cap \tilde{g}$ и $\alpha \neq \mu_{i}, \mu_{j}$, то найдется такая $b \in B$, что $\alpha \in b$, но тогда $\hat{b}$ есть одна или две точки, лежашие на $g$ между $\nu_{i}$ и $\nu_{i+1}$, а значит равные им (или одной из них); отсюда $\hat{g}=b \subset A$, что противоречит п. 1.

3. Из п. 2 следует, что $\tilde{f}$ и $\tilde{g}$ образуют вместе простую замкнутую ломаную $l$, ограничивающую некоторый односвязный многоугольник $\widetilde{M} \subset M$.

4. Заметим, что найдется $k>i+1$ такое, что $\mu_{i+1}=\nu_{k}$, но тогда $\nu_{k} \in \widetilde{M}$, а значит, часть $g$, начинающаяся в $\nu_{i+1}$, и до конца вся лежит в $\widetilde{M}$, что противоречит условию совпадения конщов $f$ и $g$, а значит, исходное допущение неверно и лемма доказана.

Лемма 2.2. Пусть $f, g, M$ как в лемме 2.1 и существуют открытые множества $U$ и $V$ такие, что $U \ni$ начало $f$ и $g$; $V \ni$ конеи $f u g ; U \cap f(F)=U \cap g(G)$; $V \cap f(F)=V \cap g(G)$. Тогда $\operatorname{tw}(f)=\operatorname{tw}(g)$.

ДоКАЗАТЕЛЬСтво. Заметим, что из леммы 2.1 следует, что $\mu=\nu$, а отсюда с помощью индукции по $\mu_{k}$ нетрудно убедиться в справедливости утверждаемого.

ЛЕмма 2.3. Пусть $(T, \tau)$ - выпуклое вложение дерева, $p, q \in \delta_{\tau} T, p \neq q$. Тогда $\operatorname{agg}_{\tau}(p, q) \leqslant 0$.

ДокАЗАТЕЛьство. Прежде всего введем некоторые обозначения:

- $e_{p q}$ - ребро ломаной $T^{p q}$, инщидентное вершине $p$;

- $M$ - вьпуклая оболочка $\tau(T)$;

- $W$ - та из двух дуг $\partial M$, образованных образами вершин $p$ и $q$, ориентированных от $\tau(p)$ к $\tau(q)$, tw которой больше;

- $w_{p}^{p q}, w_{q}^{p q}$ - непересекающиеся отрезки, включенные в $W$, инцидентные $\tau(p)$ и $\tau(q)$ соответственно.

Заметим, что если $L$ - прямая, натянутая на отрезок $w_{p}^{p q}$, то пересечения отрезков $w_{p}^{p q}, \tau\left(e_{1}^{p}\right), \tau\left(e_{p q}\right)$ с окружностью достаточно малого радиуса с центром в $\tau(p)$ лежат по одну сторону относительно прямой $L$ и угловые координаты этих точек пересечения монотонно возрастают. Отсюда ясно, что все взаиморасположения отрезков $w_{p}^{p q}, \tau\left(e_{1}^{p}\right)$, $\tau\left(e_{p q}\right)$ могут быть такими:

1) $w_{p}^{p q} \neq \tau\left(e_{1}^{p}\right) \neq \tau\left(e_{p q}\right)$, и следовательно, $w_{p}^{p q} \neq \tau\left(e_{p q}\right)$;

2) $w_{p}^{p q} \neq \tau\left(e_{1}^{p}\right)=\tau\left(e_{p q}\right)$;

3) $w_{p}^{p q}=\tau\left(e_{1}^{p}\right) \neq \tau\left(e_{p q}\right)$;

4) $w_{p}^{p q}=\tau\left(e_{1}^{p}\right)=\tau\left(e_{p q}\right)$,

и аналогичное замечание можно сделать об отрезках $w_{q}^{p q}, \tau\left(e_{2}^{q}\right), \tau\left(e_{q p}\right)$, однако здесь угловые координаты будут убывать.

Определим еще три простые ломаные:

- $X=$ расширение $\tau$-образа $T^{p q}$ ребрами $w_{p}^{p q}, w_{q}^{p q}$;

- $Y=$ дополнительная к $W$ дуга, расширенная $w_{p}^{p q}, w_{q}^{p q}$;

- $Z$ = та же дуга, расширенная $\tau$-образами $e_{1}^{p}, e_{2}^{q}$.

С помощью леммы 2.2 , замечания и ломаных $X, Y, Z, \widehat{T}^{p q}$ нетрудно убедиться, что для каждой из пар случаев 1-4 для $p, q$ выполняется утверждаемое неравенство.

Для завершения доказательства теоремы 1.1 определим $r_{p q}^{p}, r_{p q}^{q}$ как лучи с началами в $\tau(p)$ и $\tau(q)$, содержашие интервалы $\tau\left(e_{1}^{p}\right)$ и $\tau\left(e_{2}^{q}\right)$ соответственно. 
Лемма 2.4. Пусть $(T, \tau)$ - выпуклое вложсение дерева, $p, q \in \delta_{\tau} T, p \neq q$, $\operatorname{agg}_{\tau}(p, q)=0$. Тогда лучи $r_{p q}^{p}, r_{p q}^{q}$ лежсат на одной прямой $l_{p q}$ икажсдий из них в пересечении с ӘМ образует невырожденный отрезок.

ДокАЗАТЕЛЬСТвО. Из рассмотрения случаев взаиморасположения, описанных в доказательстве леммы 2.3, непосредственно вытекает утверждаемое.

Лемма 2.5. Пусть $(T, \tau)$ - выпуклое вложсние дерева, $p, q \in \delta_{\tau} T, p \neq q$. Тогда если хоть одно из ребер $e_{1}^{p}$ или $e_{2}^{q}$ двустороннее, $\operatorname{mog}_{\tau}(p, q) \neq 0$.

ДокАЗАтЕльство. Допустим $\operatorname{agg}_{\tau}(p, q)=0$. Тогда из леммы 2.4 вытекает, что вся conv $\delta_{\tau} T$ лежит по одну сторону относительно $l_{p q}$, что противоречит наличию двустороннего ребра.

Таким образом теорема 1.1 доказана.

3. О достаточности необходимого условия выпуклой реализуемости для случая ломаных. В этом разделе доказьвается теорема 1.2.

Мы не будем отличать в этом разделе ломаные от простых ломаных, и будем считать, что ломаная параметризована деревом, вершины которого суть $0, \ldots, n+1$, а ребра суть пары $\{0,1\}, \ldots,\{n, n+1\}$, а также, что у ломаных нет фиктивных вершин.

Прежде всего сформулируем несколько определений.

Назовем конечную последовательность чисел $\varphi=\left(\varphi_{1}, \ldots, \varphi_{n}\right)$ угловой последовательностью, если найдется ориентированная ломаная $f$ такая, что покомпонентно совпадают наборы чисел $\operatorname{ang}_{f}$ и $\varphi$ (ясно, что это равносильно условию $\varphi_{i} \in(-\pi, 0) \cup(0, \pi)$, где $i=1, \ldots, n)$.

Пусть $\varphi-$ угловая последовательность длины $n$. Тогда определим ее расширение $\bar{\varphi}_{0}, \ldots, \bar{\varphi}_{n+1}$, положив

$$
\bar{\varphi}_{i}= \begin{cases}\varphi_{i}, & i=1, \ldots, n \\ -\pi \operatorname{sign} \varphi_{1}, & i=0, \\ -\pi \operatorname{sign} \varphi_{n}, & i=n+1 .\end{cases}
$$

Для $\varphi$ - угловой последовательности длины $n$, и $i, j=0, \ldots, n+1$, где $i \leqslant j$, обозначим $\varphi(i, j):=\sum_{k=i}^{j} \bar{\varphi}_{k}$.

Угловую последовательность $\varphi$ длины $n$ назовем выпуклой, если $\forall i, j=0, \ldots, n+1$, $i<j, \operatorname{sign} \bar{\varphi}_{i}=\operatorname{sign} \bar{\varphi}_{j}=: \varepsilon$ вьполняется

1) $\varphi(i, j) \in[0,2 \pi \varepsilon]$;

2) если $i>1$ или $j<n$, то $\varphi(i, j) \neq 2 \pi \varepsilon$;

3) если $\operatorname{sign} \varphi_{i-1}$ или $\operatorname{sign} \varphi_{j+1}=-\varepsilon$, то $\varphi(i, j) \neq 0$.

Заметим, что при этом из второго условия вытекает, что в третьем условии $\varphi(i, j) \neq$ $2 \pi \varepsilon$.

Подмножество $S$ множества целых чисел $\mathbb{Z}$ назовем связным, если оно является пересечением некоторого отрезка в $\mathbb{R} \mathrm{c} \mathbb{Z}$.

Если $\varphi$ - угловая последовательность длины $n$, то множество $S \subset\{1, \ldots, n\}$ назовем $\varphi$-знакопостоянным, если $\varphi$ имеет постоянньй знак на нем.

Максимальное по включению связное $\varphi$-знакопостоянное множество назовем $\varphi$-сегментом.

Ясно, что всякий $\varphi$-сегмент не пуст. Определим знак $\varphi$-сегмента, как знак $\varphi$ на нем. Легко видеть, что для всякой угловой последовательности $\varphi$ найдется одна и только 
одна конечная естественно упорядоченная последовательность $\Gamma_{1}^{\varphi}, \ldots, \Gamma_{m}^{\varphi}$ всех ее $\varphi$-сегментов, где $m$ - их количество, причем видно, что знаки сегментов чередуются.

Обозначим через $\delta f$-множество всех вершин ломаной $f$ и назовем границей $f$ (ясно, что эта граница совпадает с границей, определенной во введении); через $M_{f}$ - выпуклую оболочку границы $\delta f$ ломаной $f$.

Назовем ломаную $g$ подломаной в ломаной $f$, если $g$ - подграфф в $f$.

Ясно, что если $f$ - ломаная, то она лежит в $M_{f}$, а если $f$ выпукла, то $\delta f \subset \partial M_{f}$.

Если $f$ - невырожденная (т.е. длины большей нуля) выпуклая ломаная, то $\partial M_{f}$ гомеоморфна окружности $S^{1}$, а $\partial M_{f}$ без двух концевых вершин ломаной состоит из двух “дуг", т.е. связных компонент, гомеоморфных прямой $\mathbb{R}$. Если точка $x \in \partial M_{f}-$ не концевая вершина $f$, то обозначим через $W_{x}^{f}$ ту из этих дуг, которая содержит $x$.

Ясно, что если $f$ - выпуклая ломаная, а $g$ - ее подломаная, то $g$ выпукла.

Заметим, что если $f$ - ориентированная невырожденная выпуклая ломаная, то знаки всяких двух ее вершин совпадают, если и только если совпадают их дуги.

Лемма 3.1. Пусть $f$ - ориентированная выпуклая невырожсденная ломаная. Тогда $\operatorname{ang}_{f}-$ выпуклая угловая последовательность.

ДокАЗАТЕЛьСТво. Пусть длина $f$ равна $n$.

1. Рассмотрим случай, когда $i, j \in\{1, \ldots, n\}, i<j, \operatorname{sign}_{f}(i)=\operatorname{sign}_{f} \operatorname{ang}_{f}(j)=: \varepsilon$.

Без ограничения общности положим $\varepsilon=-1$. Заметим, что $0 \geqslant \operatorname{agg}_{f}(i, j)=\operatorname{ang}_{f}(i, j)$. Если sign $\operatorname{ang}_{f}(i-1)$ или sign $\operatorname{ang}_{f}(j+1)=1$, то, по крайней мере, одно из ребер $\{i-1, i\}$ или $\{j, j+1\}$ двустороннее, а значит, неравенство строгое (см. теорему 1.1). Нетрудно увидеть, что $\operatorname{agg}_{f}(j+1, i-1)=-\operatorname{ang}_{f}(i, j)+\lambda$, где $\lambda$ - число, составленное из слагаемых, входящих в agg в вершинах $i-1$ и $j+1$; оно может принимать значения из $[-2 \pi, 0]$, что вместе с теоремой 1.1 влечет $\operatorname{ang}_{f}(i, j) \geqslant-2 \pi$. Eсли sign ang $f(i-1)=1$, то $\lambda=-\operatorname{ang}_{f}(i, j)+\varkappa$ для некоторого $\varkappa \in[-\pi, 0]$. Следовательно, $\lambda>-2 \pi$ и $\operatorname{ang}_{f}(i, j)>$ $-2 \pi$. Если же sign $\operatorname{ang}_{f}(i-1)=-1$, то $-2 \pi \leqslant \operatorname{ang}_{f}(i-1, j)=\operatorname{ang}_{f}(i, j)+\operatorname{ang}_{f}(i-1)$, что влечет $\operatorname{ang}_{f}(i, j) \geqslant-2 \pi-\operatorname{ang}_{f}(i-1)>-2 \pi$.

2. Случаи $i=0, j \in\{1, \ldots, n\} ; i \in\{1, \ldots, n\}, j=n+1 ; i=0, j=n+1$, рассматриваются аналогично.

Таким образом лемма доказана.

Далее будем считать, что задана вьпуклая угловая последовательность $\varphi$ числа сегментов $m>0$.

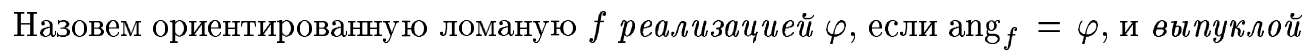
реализачией, если к тому же $f$ выпукла.

Пусть $k=0, \ldots, m$; тогда назовем ориентированную ломаную $f$ выnуклой $k$-peaлизацией $\varphi$, если $f$ удовлетворяет следующим условиям:

- при $k=0 f$-произвольная ориентированная вырожденная ломаная;

- при $k>0 f$ - выпуклая ориентированная ломаная такая, что $\operatorname{ang}_{f}$ совпадает с первыми $k$ сегментами $\varphi$.

Если $f$ - ориентированная ломаная, то определим для нее направления:

- $H_{f}$ - единичный вектор, сонаправленный последнему ребру $f$;

- $G_{f}$ - единичный вектор такой, что если $f$ невырождена, то он сонаправлен предпоследнему ребру $f$, а если $f$ вырождена, то $G_{f}=-H_{f}$. 
Если $f$ - вьпуклая ориентированная ломаная, то $x_{f}, y_{f}$ - векторы единичной длины такие, что если $f$ вырождена, то $x_{f}=H_{f}, y_{f}=G_{f}$; иначе $x_{f}$ совпадает с вектором $H$ для ориентированной ломаной, образованной естественно ориентированной дугой $\partial M_{f}$ без конщов $f$, не содержащей последнего ребра $f$; обозначив через $g$ ломаную $f$ без последнего ребра, определим вектор $y_{f}$ как вектор $H$ для ориентированной ломаной, образованной той естественно ориентированной дугой $\partial M_{g}$ без конщов $g$, которая содержится в дуге $\partial M_{f}$, дополняющей дугу, образующую $x_{f}$.

Определим для $\varphi$ последовательность

$$
s_{k}= \begin{cases}\operatorname{sign} \Gamma_{k}^{\varphi}, & k=1, \ldots, m, \\ -\operatorname{sign} \Gamma_{1}^{\varphi}, & k=0 .\end{cases}
$$

Будем рассматривать вместе с вьпуклой $k$-реализацией $f$ угловой последовательности $\varphi$ числа

$$
\begin{aligned}
& q= \begin{cases}1+\max \Gamma_{k}^{\varphi}, & k=1, \ldots, m, \\
1, & k=0,\end{cases} \\
& s=\max \Gamma_{k+1}^{\varphi}, \quad k=0, \ldots, m-1 .
\end{aligned}
$$

Назовем подходящей $k$-реализачией $\varphi$ ломаную $f$, являющуюся выпуклой $k$-реализацией $\varphi$, и такую, что если $k<m$, то выполнены следующие четыре условия:

1) для всякого $j \geqslant q$ такого, что $\bar{\varphi}_{q} \bar{\varphi}_{j}>0$, верно

$$
s_{k} \angle\left(H_{f}, x_{f}\right) \geqslant s_{k} \varphi(q, j)
$$

$2)$ для всякого $j \geqslant q-1$ такого, что $\bar{\varphi}_{q-1} \bar{\varphi}_{j}>0$ верно

$$
s_{k} \angle\left(G_{f}, y_{f}\right) \leqslant s_{k} \varphi(q-1, j)
$$

3 ) для всякого $j \geqslant q$ такого, что $\bar{\varphi}_{q} \bar{\varphi}_{j}>0$ и сегмент, содержащий $j$, состоит только из $j$, верно

$$
s_{k} \angle\left(H_{f}, x_{f}\right)>s_{k} \varphi(q, j)
$$

$4)$ для всякого $j \geqslant q-1$ такого, что $\bar{\varphi}_{q-1} \bar{\varphi}_{j}>0$ и сегмент (если $\left.j>q-1\right)$, содержащий $j$, состоит только из $j$, верно

$$
s_{k} \angle\left(G_{f}, y_{f}\right)<s_{k} \varphi(q-1, j) .
$$

\section{ЛЕмма 3.2. Существует подходящая 0-реализация.}

ДокАЗАТЕЛЬСТво. Возьмем произвольную вырожденную ориентированную ломаную $f$. Она выпукла и, следовательно, чтобы доказать, что она подходящая 0-реализация, достаточно доказать неравенства из 1)-4). Без ограничения общности будем считать, что $s_{0}=1$.

Первое неравенство $\angle\left(H_{f}, x_{f}\right) \geqslant \varphi(q, j)$, где $q=1$, выполняется, ибо $\angle\left(H_{f}, x_{f}\right)=0$ (так как $\left.H_{f}=x_{f}\right)$, а $\varphi(q, j) \leqslant 0$ по вьпуклости $\varphi$.

Второе неравенство $\angle\left(G_{f}, y_{f}\right) \leqslant \varphi(q-1, j)$ выполняется, ибо $G_{f}=y_{f}$ и $\varphi(q-1, j) \geqslant 0$ по выпуклости $\varphi$.

Неравенства из 3) и 4) точно так же непосредственно следуют из определений. Лемма доказана.

Если $f$ - подходящая $k$-реализация $\varphi, k<m$, то определим $\mu=\frac{1}{2}(\alpha+\beta)$, где

$$
\begin{aligned}
& \beta=s_{k} \min \{|\varphi(q-1, j)|: j \geqslant q-1, \bar{\varphi}(q-1) \bar{\varphi}(j)>0\}, \\
& \alpha=s_{k} \max \left\{s_{k} \angle\left(G_{f}, y_{f}\right), s_{k} \varphi(q-1, s)\right\} .
\end{aligned}
$$

Это число $\mu$ будет основой продолжения $f$. 
ЛЕмма 3.3. Для $f$ - подходящей $k$-реализачии набора $\varphi, k<m$, верны следуюшие неравенства:

1) $s_{k} \mu \geqslant s_{k} \angle\left(G_{f}, y_{f}\right)$;

2) $s_{k} \mu<s_{k} \bar{\varphi}_{q-1}$;

3) $s_{k} \mu>s_{k} \varphi(q-1, s)$;

4) $s_{k} \mu \leqslant s_{k}(-\pi+\varphi(q-1, s))$.

ДокАЗАТЕЛЬСТво. Без ограничения общности будем считать, что $s_{k}=-1$. Тогда

$$
\mu=\frac{1}{2}\left(\min \left\{\angle\left(G_{f}, y_{f}\right), \varphi(q-1, s)\right\}+\max \left\{\varphi(q-1, j): j \geqslant q-1, \bar{\varphi}_{j}<0\right\}\right) .
$$

Докажем 1). Ясно, что $\mu \leqslant \frac{1}{2}\left(\angle\left(G_{f}, y_{f}\right)+\beta\right)$. Вычислим ограничение сверху на $\beta$. Из второго условия в определении подходящей $k$-реализации следует, что $\angle\left(G_{f}, y_{f}\right) \geqslant$ $\varphi(q-1, j), j \geqslant q-1$, при $\bar{\varphi}_{q-1} \bar{\varphi}_{j}>0$, т.е. $\beta \leqslant \angle\left(G_{f}, y_{f}\right)$, а значит, $\mu \leqslant \angle\left(G_{f}, y_{f}\right)$.

Докажем 2). Оценим $\beta$ снизу:

$$
\beta=\max \left\{\varphi(q-1, j): j \geqslant q-1, \bar{\varphi}_{j}<0\right\} \geqslant \varphi(q-1, q-1)=\bar{\varphi}_{q-1} .
$$

Оценим снизу $\alpha$, т.е. оценим $\angle\left(G_{f}, y_{f}\right)$ и $\varphi(q-1, s): \angle\left(G_{f}, y_{f}\right)>\bar{\varphi}_{q-1}$ из четвертого условия определения подходящей $k$-реализации и

$$
\varphi(q-1, s)=\bar{\varphi}_{q-1}+\varphi_{q}+\cdots+\varphi_{s}>\bar{\varphi}_{q-1}
$$

так как $\varphi_{q}+\cdots+\varphi_{s}>0$, из-за того, что знаки сегментов чередуются.

Из этих оценок следует, что $\mu>\bar{\varphi}_{q-1}$.

Докажем 3). Допустим противное, т.е. $\varphi(q-1, s) \leqslant \mu$. Тогда из первого свойства вытекает, что $\varphi(q-1, s) \leqslant \mu \leqslant \angle\left(G_{f}, y_{f}\right)$, и $\mu=\frac{1}{2}(\varphi(q-1, s)+\beta)$. Из последних двух неравенств и доказательства второго свойства видно, что $\beta>\varphi_{q-1}$, и найдется $j>q-1$ такое, что $\bar{\varphi}_{j}<0$ и $\varphi(q-1, s)+\varphi(s+1, j)=\varphi(q-1, j) \geqslant \varphi(q-1, s)$. Заметим, что отсюда $\varphi(s+1, j) \geqslant 0$. Рассмотрим два случая:

$1)$ если $s+1=j$, то $\varphi(s+1, s+1)=\bar{\varphi}_{s+1} \geqslant 0$, что противоречит тому, что $\bar{\varphi}_{s+1}<0$, так как $s+1$ следует непосредственно за положительным сегментом $\Gamma_{k+1}^{\varphi}$;

$2)$ если $s+1<j$, то $\varphi(s+1, j) \in[-2 \pi, 0)$ из третьего условия определения выпуклой угловой последовательности, что также противоречиво.

Докажем 4). Из второго условия определения подходящей $k$-реализации следует, что $\angle\left(G_{f}, y_{f}\right) \geqslant \beta$. Заметим, что $\varphi(q-1, s) \geqslant \beta$, так как $\beta=\max \left\{\bar{\varphi}_{q-1}, \varphi(q-1, s)+\gamma\right\}$, где $\gamma=\max \left\{\varphi(s+1, j): j \geqslant s+1, \bar{\varphi}_{s+1} \bar{\varphi}_{j}>0\right\}$, и из первого условия определения выпуклой угловой последовательности вытекает, что $\gamma \leqslant 0$, и, как в первом пункте, $\bar{\varphi}_{q-1}<\varphi(q-1, s)$.

Отсюда следует, что $\mu \geqslant \beta$, так как $\beta \leqslant \varphi(q-1, s)$ и $\alpha$ оценено через $\beta$.

Оценим снизу $\beta: \beta \geqslant \varphi(q-1, s+1)=\varphi(q-1, s)+\bar{\varphi}_{s+1} \geqslant \varphi(q-1, s)+(-\pi)$.

Таким образом лемма доказана.

Из леммы 3.3 следует, что $\mu \in(-\pi, \pi)$, а значит, может считаться ориентированным углом между некоторыми векторами. Определим вектор $Z_{f, \varphi}$ как вектор единичной длины такой, что $\angle\left(G_{f}, Z_{f, \varphi}\right)=\mu$.

С помощью вектора $Z_{f, \varphi}$ построим продолжение $f$.

Нетрудно показать, что верно следующее утверждение. 
Лемма 3.4. Пусть числа $\phi_{0}, \ldots, \phi_{n}, \mu \in \mathbb{R}, \quad n=1,2, \ldots$, таковь, что $0=\phi_{0}<$ $\phi_{1}<\cdots<\phi_{n} \leqslant \mu+\pi ; 0<\mu \leqslant \pi ; \phi_{i}-\phi_{i-1}<\pi, i=1, \ldots, n ; \mu<\phi_{n}$.

Тогда числа $\psi_{1}, \ldots, \psi_{n}$, индуктивно определенные равенствами

$$
\psi_{n}=\mu, \quad \psi_{i}=\frac{1}{2}\left(\max \left\{0, \phi_{i+1}-\pi\right\}+\min \left\{\phi_{i}, \psi_{i+1}\right\}\right), \quad i=n-1, \ldots, 1,
$$

обладают следующими свойствами:

$$
\begin{gathered}
0<\psi_{1}<\cdots<\psi_{n-1} ; \quad \psi_{i}<\phi_{i}, \quad i=1, \ldots, n \\
\phi_{i+1}-\psi_{i}<\pi, \quad i=1, \ldots, n-2 \\
\phi_{n}-\psi_{n-1}<\pi, \quad \psi_{n-1}<\psi_{n}, \quad \text { если } \phi_{n}<\mu+\pi \\
\psi_{n-1}=\mu, \text { если } \phi_{n}=\mu+\pi
\end{gathered}
$$

зависимость $\psi$ от $(\phi, \mu)$ непрерывная.

$\Phi$ ункцию $\Phi$, сопоставляющую угловой последовательности $\varphi$ некоторую ориентированную ломаную $f=\Phi(\varphi)$ такую, что ang $f=\varphi$, назовем непрерывной, если каждая вершина этой ломаной $f$ непрерьвно зависит от набора действительных чисел $\varphi$.

ЛЕмма 3.5. Пусть $R$ - луч на плоскости с началом в точке $c, d \in R \backslash$ $\{c\}, \quad 0<\mu \leqslant \pi$. Тогда существует непрерывная функиия $\Phi$, сопоставляющая набору чисел $\varphi_{1}, \ldots, \varphi_{n}$, где $n=1,2, \ldots$, при том, что набор чисел $\phi_{0}=0$, $\phi_{1}=\varphi_{1}, \ldots, \phi_{n}=\varphi_{1}+\cdots+\varphi_{n}, \mu$ удовлетворяет условию леммы 3.4 , ломаную $f=\Phi(\varphi)$ со свойствами

1) $\operatorname{ang}_{f}=\varphi$

2) $f_{0}=c$

3) $f_{1}=d$;

4) $\angle\left(\overrightarrow{c d}, \overrightarrow{c f_{n+1}}\right)=\mu$.

ДокАЗАТЕЛЬСТво. Проведем построение по индукции. Пусть точки $f_{0}, f_{1}, \ldots, f_{i}$ уже построены. Тогда определим $f_{i+1}$ как единственную точку пересечения лучей $\{c+$ $t h: t \geqslant 0\}$ и $\left\{f_{i}+t j: t \geqslant 0\right\}$, где $\angle(\overrightarrow{c d}, h)=\psi_{i+1}, \angle(\overrightarrow{c d}, j)=\phi_{i}$, при этом если $\phi_{n}<\mu+\pi$, то $i=1, \ldots, n$, а если $\phi_{n}=\mu+\pi$, то $i=1, \ldots, n-1$, и в качестве точки $f_{n+1}$ возьмем середину отрезка $\left[c, f_{n}\right]$.

Нетрудно убедиться, что такое построение непрерьвно.

ЛЕмма 3.6. Если $f$ - подходящая $k$-реализачия $\varphi$ и $k<m$, то найдется $g-$ выпуклая $(k+1)$-реализаиия $\varphi$.

ДокАЗАТЕЛЬСтво. Построим $g$ как продолжение $f$. Без ограничения общности можно считать, что $s_{k}=-1$. Временно переобозначим

$$
\begin{aligned}
\varphi_{i} & :=\varphi_{i+q-1}, \quad i+q-1 \in \Gamma_{k+1}^{\varphi}, \\
\mu & :=-\bar{\varphi}_{q-1}+\mu
\end{aligned}
$$

и по этим числам с помощью функции $\Phi$ из леммы 3.5 на точках $c=f_{q-1}, d=f_{q}$ пристроим к $f$ ее продолжение и получим $g$. 
Возможность применения предыдущей леммы обеспечивается леммой 3.3.

Докажем теперь, что построенное в лемме 3.6 продолжение является подходящей $(k+1)$-реализацией (отсюда видно, что можно осуществить индуктивное построение реализации угловой последовательности $\varphi$ ).

ЛЕмма 3.7. Если $f$ - подходящая $k$-реализачия $\varphi$ и $k<m$, то найдется $g$ подходящая $(k+1)$-реализация $\varphi$.

ДокАЗАТЕЛЬСТво. Возьмем ломаную $g$, построенную в лемме 3.6. По лемме 3.3 она выпукла. Поэтому осталось только проверить для нее условия 1)-4) из определения подходящей $k$-реализации, при условии, что $k+1<m$.

Заметим, что $\angle\left(H_{g}, x_{g}\right)=\mu-\varphi(q-1, s)$, и если сard $\Gamma_{k+1}^{\varphi}=1$, то $y_{g}=x_{f}$, а если $\operatorname{card} \Gamma_{k+1}^{\varphi}>1$, то ясно, что $y_{g}=G_{g}$. Без ограничения общности можно считать, что $s_{k}=-1$.

Первое условие. Оно эквивалентно $\mu \geqslant \varphi(q-1, j)$, при $j \geqslant s+1, \bar{\varphi}_{j}<0$, а это неравенство вытекает из следующих трех замечаний:

- ясно, что $\varphi(q-1, j) \leqslant \beta$;

- из вьпуклости $\varphi$ следует $\varphi(q-1, j)=\varphi(q-1, s)+\varphi(s+1, j) \leqslant \varphi(q-1, s)$;

- из второго условия определения подходящей $k$-реализации следует, что $\varphi(q-$ $1, j) \leqslant \angle\left(G_{f}, y_{f}\right)$.

Второе условие. Если саrd $\Gamma_{k+1}^{\varphi}=1$, то $\angle\left(G_{g}, y_{g}\right)=\angle\left(H_{f}, x_{f}\right) \leqslant \min \{\varphi(s, j): j \geqslant$ $\left.s=q, \bar{\varphi}_{j}>0\right\}$ из того, что $f-$ подходящая $k$-реализация.

Иначе $\angle\left(G_{g}, y_{g}\right)=0 \leqslant \min \left\{\varphi(s, j): j \geqslant s, \bar{\varphi}_{j}>0\right\}$ из вьпуклости $\varphi$.

Третье условие. Пусть $j \in \Gamma_{\nu}^{\varphi}, \nu=k+2, k+4, \cdots<m, \operatorname{card} \Gamma_{\nu}^{\varphi}=1$. Тогда сравним $\mu-\varphi(q-1, s) \mathrm{c} \varphi(s+1, j)$, т.е. $\mu \mathrm{c} \varphi(q-1, j)$. Из определения $\mu$ и того, что $f-$ подходящая $k$-реализация (см. условие 4$))$ легко видеть, что это сравнение эквивалентно сравнению 0 c $\varphi(s+1, j)$.

Если $s+1=j$, то $0>\varphi_{s+1}$ из определения угловой последовательности, а если $j>s+1$, то $0>\varphi(s+1, j)$ из выпуклости $\varphi$.

Четвертое условие. 1 . Если $j=s$, то $\angle\left(G_{g}, y_{g}\right)$ сравним с $\varphi_{s}$ : если $s=q$, то $y_{g}=x_{f}$, и из того, что $f$ - подходящая $k$-реализация $\varphi$, следует, что $\angle\left(H_{f}, x_{f}\right)=\angle\left(G_{g}, y_{g}\right)<\varphi_{q}$; если $s>q$, то $y_{g}=G_{g}$, т.е. надо сравнить 0 и $\varphi_{s}$, но $0<\varphi_{s}$, так как $s_{k}=-1$.

2. Пусть теперь $j \in \Gamma_{\nu}^{\varphi}, \nu=k+3, k+5, \ldots<m, \operatorname{card} \Gamma_{\nu}^{\varphi}=1$. Тогда надо сравнить $\angle\left(G_{g}, y_{g}\right)$ с $\varphi(s, j)$. Если $s=q$, то $y_{g}=x_{f}$, и по третьему условию определения подходящей $k$-реализации верно $\angle\left(H_{f}=G_{g}, x_{f}\right)<\varphi(q, j)$. Если $s>q$, то $y_{g}=G_{g}$, и тогда надо сравнить 0 с $\varphi(s, j)$. Из вьпуклости $\varphi$ следует, что $0<\varphi(s, j)$ (см. третье условие определения выпуклости). Таким образом лемма доказана.

Итак, мы пришли к основному результату этого раздела.

ТЕОРемА 3.1. Плоская ломаная длины $n \geqslant 1$ выпукло реализуема, если и только если ее угловая последовательность ( с какой-нибудь, а следовательно, и с противополохной, ориентацией) является выпуклой.

Существует такой алгоритм построения выпуклой реализации выпуклой угловой последовательности, ито функиия, соответствующая этому алгоритму, непрерывна.

Кроме того, все выпуклые угловые последовательности данной длины $n$ образуют многогранное мнохество, состоящее из $2^{n}$ связых компонент, кахдая из 
которых выпукла; а если $n \geqslant 3$, то замыкание всего многогранника связно и не выпукло.

ДокАЗАтЕЛьство. Необходимость была доказана в лемме 3.1. Достаточность следует из лемм 3.2 и 3.7 , дающих соответственно базу и шаг индукции. То, что выпуклые угловые последовательности длины $n$ образуют многогранное множество $J$, следует из того, что они определяются системой линейных неравенств. Количество связных компонент и их вьпуклость вытекает из определения, а то, что замыкание $J$ при $n \geqslant 3$ не выпукло, следует из того, что всегда найдутся две выпуклые угловыепоследовательности длины $n$ такие, что соединяющий их отрезок не лежит в $J$. Приведем пример таких последовательностей:

$$
\begin{gathered}
a=\left(\frac{\pi}{4}, \frac{\pi}{4},-\frac{\pi}{4},-\frac{\pi}{2}, \frac{\pi}{2},-\frac{\pi}{2}, \ldots,(-1)^{k-1} \frac{\pi}{2}, \ldots,(-1)^{n-1} \frac{\pi}{2}\right), \quad \text { где } k \geqslant 4, \\
b=\left(-\frac{\pi}{4}, \frac{\pi}{2},-\frac{\pi}{4},-\frac{\pi}{2}, \frac{\pi}{2},-\frac{\pi}{2}, \ldots,(-1)^{k-1} \frac{\pi}{2}, \ldots,(-1)^{n-1} \frac{\pi}{2}\right), \quad \text { где } k \geqslant 4 .
\end{gathered}
$$

Нетрудно убедиться, что эти последовательности вьпуклы. А если взять $c=a t+$ $b(1-t)$, где $0<t<1 / 2$, то $c$ не выпукла, так как

$$
c_{1}+c_{2}+c_{3}=-\frac{\pi}{4}+t \frac{\pi}{2}+\frac{\pi}{2}-t \frac{\pi}{4}-\frac{\pi}{4}=t \frac{\pi}{4}>0
$$

что противоречит первому условию определения выпуклой угловой последовательности.

Автор благодарен профессору А. О. Иванову и профессору А. А. Тужилину за постоянное внимание к работе.

\section{СПИСОК ЦИТИРОВАННОЙ ЛИТЕРАТУРЫ}

[1] Fomenko A. T., Tuzhilin A. A. Elements of Geometry and Topology of Minimal Surfaces in Three-Dimensional Space // Transl. Math. Monographs. 1992. V. 93.

[2] Иванов А. О., Тужилин А. А. Геометрия минимальных сетей и одномерная проблема Плато // УМН. 1992. Т. 47. №2(284). С. 53-115.

[3] Ivanov A. O., Tuzhilin A. A. Minimal Networks. Steiner Problem and Its Generalizations. CRC Press, 1994.

[4] Melzak Z. A. On the problem of Steiner // Canad. Math. Bull. 1960. V. 4. P. 143-148.

[5] Garey M. R., Johnson D. S. The Rectilinear Steiner Problem is NP-Complete // SIAM J. Appl. Math. 1977. V. 32. P. 826-834.

[6] Иванов А. О., Тужилин А. А. Число вращения плоских линейных деревьев // Матем. сб. 1996. Т. 187. № 8. С. 41-92.

[7] Иванов А. О., Тужилин А. А. Задача Штейнера для выпуклых границ или плоские минимальные сети // Матем. сб. 1991. Т. 182. № 12. С. 1813-1844.

Московский государственный университет им. М. В. Ломоносова 